\title{
Genome-wide identification, expression, and sequence analysis of CONSTANS-like gene family in cannabis reveals a potential role in plant flowering time regulation
}

Gen Pan ${ }^{1,2+}$, Zheng $\mathrm{Li}^{1 \dagger}$, Ming Yin ${ }^{1}$, Siqi Huang ${ }^{1,2}$, Jie Tao ${ }^{1}$, Anguo Chen ${ }^{1,2}$, Jianjun Li ${ }^{1,2}$, Huijuan Tang ${ }^{1,2}$, Li Chang ${ }^{1,2}$, Yong Deng ${ }^{1,2}$, Defang $\mathrm{Li}^{1,2^{*}}$ and Lining Zhao ${ }^{1,2^{*}}$

\begin{abstract}
Background: Cannabis, an important industrial crop, has a high sensitivity to photoperiods. The flowering time of cannabis is one of its important agronomic traits, and has a significant effect on its yield and quality. The CONSTANS-like (COL) gene plays a key role in the regulation of flowering in this plant. However, the specific roles of the COL gene family in cannabis are still unknown.

Results: In this study, 13 CSCOL genes were identified in the cannabis genome. Phylogenetic analysis implied that the CsCOL proteins were divided into three subgroups, and each subgroup included conserved intron/exon structures and motifs. Chromosome distribution analysis showed that 13 CsCOL genes were unevenly distributed on 7 chromosomes, with chromosome 10 having the most CSCOL members. Collinearity analysis showed that two syntenic gene pairs of CsCOL4 and CSCOL11 were found in both rice and Gossypium raimondii. Of the 13 CsCOL genes, CsCOL6 and CsCOL12 were a pair of tandem duplicated genes, whereas CsCOL8 and CsCOL11 may have resulted from segmental duplication. Furthermore, tissue-specific expression showed that $10 \mathrm{CSCOL}$ genes were preferentially expressed in the leaves, 1 CsCOL in the stem, and 2 CsCOL in the female flower. Most CsCOL exhibited a diurnal oscillation pattern under different light treatment. Additionally, sequence analysis showed that CsCOL3 and CsCOL7 exhibited amino acid differences among the early-flowering and late flowering cultivars.

Conclusion: This study provided insight into the potential functions of CSCOL genes, and highlighted their roles in the regulation of flowering time in cannabis. Our results laid a foundation for the further elucidation of the functions of $\mathrm{COL}$ genes in cannabis.
\end{abstract}

Keywords: Cannabis, Genome-wide, CONSTANS-like gene (COL), Expression pattern, Flowering time

\footnotetext{
*Correspondence: chinakenaf@126.com; csbtzln@163.com

${ }^{\dagger}$ Gen Pan and Zheng Li contributed equally to this work.

${ }^{1}$ Institute of Bast Fiber Crops, Chinese Academy of Agricultural Sciences,

Changsha 410205, China

Full list of author information is available at the end of the article
}

C C The Author(s). 2021 Open Access This article is licensed under a Creative Commons Attribution 4.0 International License, which permits use, sharing, adaptation, distribution and reproduction in any medium or format, as long as you give appropriate credit to the original author(s) and the source, provide a link to the Creative Commons licence, and indicate if changes were made. The images or other third party material in this article are included in the article's Creative Commons licence, unless indicated otherwise in a credit line to the material. If material is not included in the article's Creative Commons licence and your intended use is not permitted by statutory regulation or exceeds the permitted use, you will need to obtain permission directly from the copyright holder. To view a copy of this licence, visit http://creativecommons.org/licenses/by/4.0/ The Creative Commons Public Domain Dedication waiver (http://creativecommons.org/publicdomain/zero/1.0/) applies to the data made available in this article, unless otherwise stated in a credit line to the data. 


\section{Background}

Hemp (Cannabis sativa L.) is an ancient economic crop that is widely used in textiles, food, and building materials, as well as other fields [1]. In recent years, the use of cannabidiols, represented by cannabinoid (CBD), has been expanding continuously, and the cannabis industry has demonstrated good prospects for development in the future [2]. Hemp is an annual short-day crop that is sensitive to photoperiods [3]. Cannabis cultivars naturally grow in high-latitude areas. However, cannabis germplasms have been introduced to low-latitude areas for planting, resulting in an early-flowering time. As a result, the growth period has been shortened, seriously reducing the yield and content of CBD and fibre [3]. Thus, the development of cannabis varieties with a wide adaptability is one of the main goals of current cannabis breeding programs. Identifying the regulatory mechanism of cannabis flowering could provide a theoretical foundation for the cultivation of cannabis varieties. However, studies on the regulatory mechanism of flowering in cannabis are currently lacking.

The flowering period of plants is a complex quantitative trait that is comprehensively regulated by many internal and external factors, including the photoperiod, temperature, hormones, and self-development [4]. Among these factors, the photoperiod is an important regulatory factor of the floral transition. In agriculture, the flowering time of cultivated plants can be adjusted to meet consumer demand by changing the length of exposure to light. With rapid advances in the fields of molecular genetics and molecular biology, many genes related to the photoperiod pathway have been discovered and cloned $[5,6]$. Studies have shown that the CONSTANS-like (COL) genes are important regulators of the plant response to photoperiods and is a core element in the regulation of plant flowering [7-9]. COL belongs to the zinc finger transcription factor family, which contains a B-box-type and a CCT (CO, CO-LIKE, TOC1) domain [10]. Depending on the number of Bbox and CCT domains, COL family genes can be divided into five groups [11]. In previous reports, the $C O L$ gene family has been comprehensively studied in many plants, including Arabidopsis, rice (Oryza sativa L.), maize (Zea mays L.), Populus, radish (Raphanus sativus L.), moso bamboo (Phyllostachys heterocycla), and Lilium $\times$ formolongi $[5,6,10-14]$. The number of $C O L$ genes varies among different species. For example, among dicot plants, the $C O L$ family has 20 members in radish and 17 members in Arabidopsis, while in monocots, 16 members have been identified in rice, 19 members in maize, and 14 members in Populus [12-16].

The $C O L$ gene functions as a transcription factor in multiple growth and development pathways, and particularly in the photoperiod-mediated flowering pathway.
Some genes in this family have been found to play an important role in the light response-mediated regulation of flowering [5,17-19], with functions that differ between short-day (SD) and long-day (LD) conditions. For example, OsCOL10, OsCOL13, and OsCOL16 function as negative regulators of flowering under both SD and LD condition in rice, while $H d 1$, a member of the $C O L$ gene family, promotes flowering under SD and suppresses flowering under LD [5, 17, 20, 21]. In Arabidopsis, the overexpression of AtCOL3, AtCOL7, and AtCOL8 can delay flowering time, while, in contrast, the overexpression of the AtCOL5 gene promotes flowering by enhancing the expression of FLOWERING LOCUS T $(F T)[18,19,22,23]$. Similar to their functions, the expression patterns also vary among the members of the COL gene family. In bananas (Musa nana Lour.), $\mathrm{MaCOL}$ genes display higher expression in light than in darkness, reaching their peak during light periods [24]. The transcript levels of PaCOL1 and PaCOL2, 2 members of the $C O L$ gene family in Norway spruce (Picea abies L.), are induced by light and increase upon transition from darkness to light [25]. Unlike PaCOL1 and PaCOL2, PttCO1 and PttCO2 showed a distinct expression pattern with an increase in expression in the early evening [26]. Meanwhile, the differences in the sequences of these genes in the CDS region were reported to associate with their functions in the photoperiodmediated flowering pathway. For example, the deletion of $2 \mathrm{bp}$ in the second exon of $H d 1$ in "Kasalath" resulted in delay of flowering time in rice accessions [20]. Similarly, nucleotide polymorphisms in the OsCOL16 coding sequence were mainly composed of three alleles (A1, A15, and A22), which varied with the flowering time [5]. These studies indicated that, due to differences in the expression patterns and CDS sequences, the COL gene family performs multiple functions in the regulation of flowering time under SD and LD conditions.

Although $C O L$ genes play an important role in the growth and development of many plants, a comprehensive analysis of the COL family genes in cannabis is currently lacking. In addition, no systemic analyses of any other gene families in cannabis have been conducted, due to the unavailability of cannabis genome assembly, with a lack of information on gene locations at the chromosome level. The genome of cannabis was recently sequenced and made available on the cannabis genomic database [27], allowing for a comprehensive analysis of the $C O L$ gene family in cannabis. In the present study, the $C O L$ gene family from cannabis was analysed using bioinformatics, and the temporal and spatial expression patterns of the $C O L$ gene were studied. Additionally, differences in the amino acid (aa) sequences of CsCOL3 and $C s C O L 7$ between early- and late-flowering cultivars were explored. Thus, the results presented in this study 
provide a biological basis for further studies to analyse the molecular functions of the $C s C O L$ gene family in cannabis.

\section{Results}

Identification of $13 \mathrm{CsCOL}$ genes in cannabis

A total of $13 \mathrm{CsCOL}$ genes were identified from the cannabis genome database (CsCOL1 through CsCOL13). The 13 CsCOL genes included both B-box and CCT conserved domains. Their physicochemical properties were analysed using ProtParam (http://web.expasy.org/ protparam/) (Table 1). As shown in Table 1, the lengths of CsCOL proteins varied from 184 (CsCOL8) to 507 (CsCOL12) aa, molecular weights ranged from 26.02 $\mathrm{kDa}$ to $56.24 \mathrm{kDa}$, and $\mathrm{pI}$ varied from 4.99 to 6.36 . In addition, the grand average of hydropathicity varied from -1.088 to -0.245 and the aliphatic index ranged from 38.10 to 69.90 (Table 1).

\section{Gene structure, phylogenetic relationship, and sequencing analysis of $\mathrm{CsCOL}$ genes}

To estimate the evolutionary relationships between the members of the $C s C O L$ gene family, we investigated the structure diversity by comparing the gene structure of the CsCOL protein. As shown in Fig. 1, all the CsCOL genes contained 2 to 5 exons and 1 to 4 introns, respectively. All of the CsCOL genes contained 3' and 5' UTR regions (Fig. 1). Furthermore, to explore the evolutionary relationships between the $C O L$ genes of different species, phylogenetic tree analysis was conducted with COL proteins from different plants, including Arabidopsis, cannabis, and rice. These included 30 genes from dicotyledonous plants (e.g., Arabidopsis and cannabis) and 14 genes from monocotyledonous plants (e.g., rice). The results revealed that these COL proteins could be clustered into three major groups, named groups I-III (Fig. 2). Group III was the smallest subfamily, which was comprised of the lowest number of COL proteins (Fig. 2). In addition, we investigated the amino acid sequence of the CsCOL genes in "Y7" and "Q1", a late- and an early-flowering varieties (Fig. S1 and Fig. S2). Unfortunately, only CsCOL3 and CsCOL7 were successfully cloned. For CsCOL3, 2 amino acid differences were found between "Y7" and "Q1", neither of which was located in a B-Box nor CCT domain (Fig. S3A). With regards to CsCOL7, 4 amino acid differences were found between "Y7" and "Q1", all of which were located in the B-BoxIdomain (Fig. S3B).

\section{Chromosomal location and synteny analysis}

As shown in Fig. 3, the $13 \mathrm{CsCOL}$ gene members were found to be unevenly distributed across 7 chromosomes of the cannabis genome, except for chromosomes 5, 6, and 7. Among these, chromosome 10 had the highest number of $C s C O L$ genes (4), while chromosomes 2, 8, and 9 only contained 1 . Interestingly, a pair of tandem replication genes were identified in chromosome 3 (CsCOL6/CsCOL12), suggesting that tandem duplication events participated in the expansion of the $C O L$ family in cannabis. As such, duplication events were investigated for the $C s C O L$ genes of the cannabis genome. As a result, only one pair of duplicated genes (CsCOL8/ CsCOL11) was identified within the cannabis genome, they may have resulted from segmental duplication or whole genome duplication (WGD) (Fig. 4). In order to further understand the evolutionary mechanism of the COL family in cannabis, collinearity diagrams of the COL family were constructed in 2 dicotyledonous plants (Gossypium raimondii and Cannabis sativa L.) and 1 monocotyledonous plant (Oryza sativa L). As shown in

Table 1 Characteristics of 13 CsCOL proteins in cannabis

\begin{tabular}{lllllll}
\hline Gene & Gene ID & Length (aa) & MW (Da) & pl & GRAVY & Aliphatic index \\
\hline CsCOL1 & LOC115722074 & 375 & $42,446.16$ & 5.82 & -0.766 & 61.63 \\
CsCOL2 & LOC115714019 & 462 & $51,911.74$ & 5.42 & -0.687 & 61.00 \\
CsCOL3 & LOC115697429 & 443 & $48,003.43$ & 5.26 & -0.563 & 61.87 \\
CsCOL4 & LOC115725326 & 387 & $41,576.35$ & 5.08 & -0.245 & 69.90 \\
CsCOL5 & LOC115714839 & 507 & $56,248.68$ & 5.95 & -0.752 & 60.95 \\
CsCOL6 & LOC115711648 & 456 & $52,718.08$ & 5.52 & -1.069 & 58.62 \\
CsCOL7 & LOC115707536 & 337 & $36,832.04$ & 5.89 & -0.555 & 64.04 \\
CsCOL8 & LOC115703699 & 184 & $20,459.75$ & 4.30 & -1.167 & 38.10 \\
CsCOL9 & LOC115700798 & 407 & $43,842.56$ & 4.89 & -0.489 & 60.96 \\
CsCOL10 & LOC115700744 & 394 & $42,808.34$ & 5.30 & -0.610 & 58.43 \\
CsCOL11 & LOC115700712 & 241 & $26,026.97$ & 4.32 & -0.741 & 55.85 \\
CsCOL12 & LOC115711183 & 420 & $48,419.95$ & 5.23 & -1.088 & 59.00 \\
CsCOL13 & LOC115700838 & 398 & $43,959.03$ & 5.19 & -0.513 & 58.84 \\
\hline
\end{tabular}




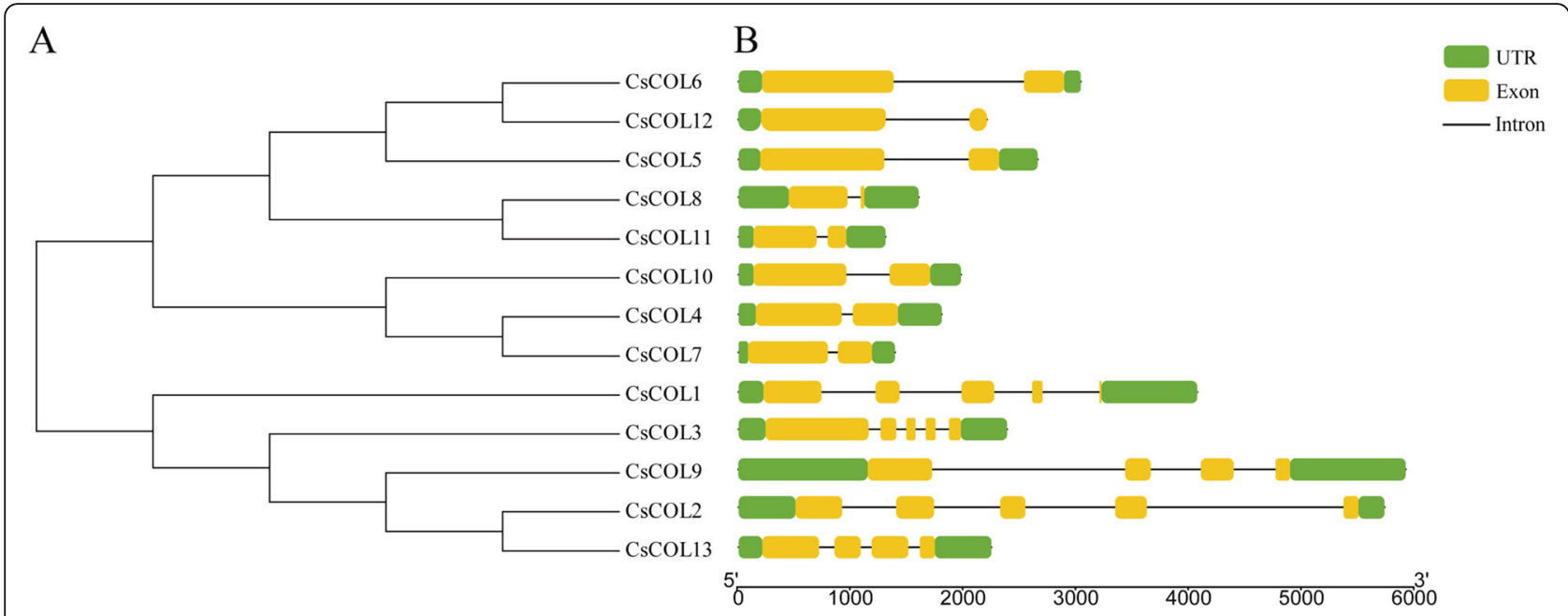

Fig. 1 Phylogenetic and gene structure analyses of cannabis CsCOL genes. a Phylogenetic analysis. b Gene structures. The exon, untranslated region (UTR), and intron are represented by the yellow and green rectangles, and a black line, respectively

Fig. 5, 15 pairs of orthologous genes were identified between cannabis and cotton Raymond, much greater than those identified between cannabis and rice (2). Among these genes, $\mathrm{CsCOL4} 4$ and $C s C O L 11$ were identified in both rice and cotton Raymond, CsCOL1, CsCOL7, CsCOL8, CsCOL5, and CsCOL9 were found in cotton Raymond alone, and the remaining were not present in any of the duplicated blocks (Fig. 5).
Spatial and temporal expression pattern analysis of 13 CsCOL genes

To gain insights into the possible role of $C s C O L$ genes in the development of cannabis, the expression pattern of the CsCOL genes was analysed in 4 plant tissues: female flower, stem, leaf, and root. The results revealed that all genes were constructively expressed in various tissues, but with different expression patterns (Fig. 6).

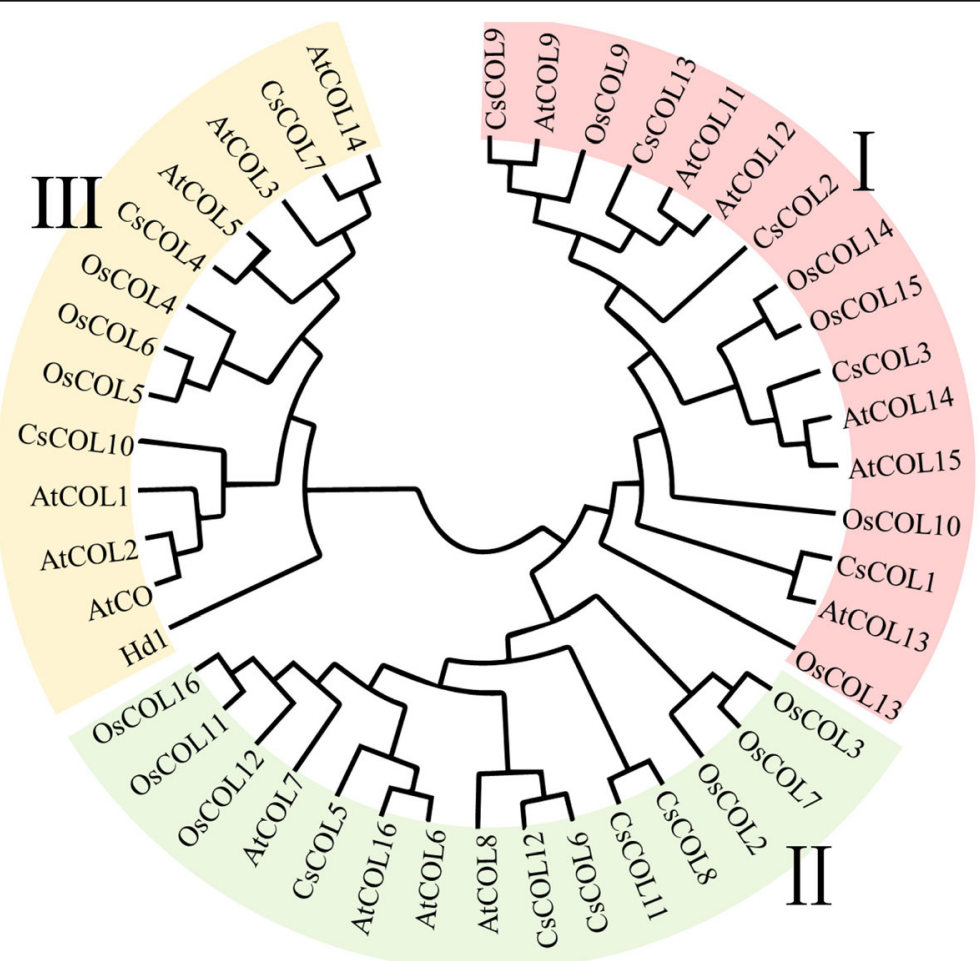

Fig. 2 Phylogenetic tree of the COL proteins from three plant species. The phylogenetic tree was constructed based on the $90 \%$ shared amino acid sites using the neighbour-joining (NJ) method. At, Arabidopsis thaliana; Os, Oryza sativa; Cs, Cannabis sativa 

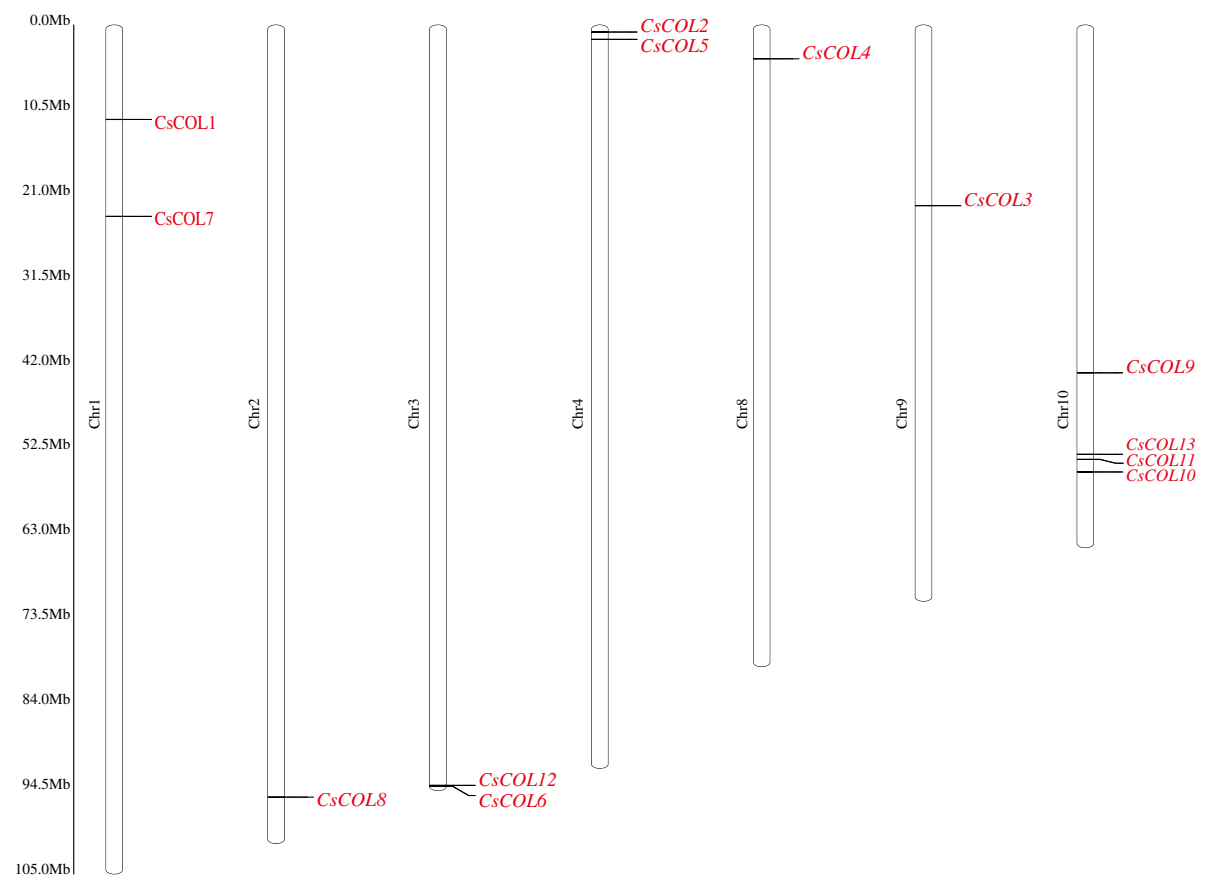

Fig. 3 The physical location of 13 CsCOL genes on cannabis chromosomes. Chromosome numbers are indicated on the left of each scaffold. Chromosome size is shown by the vertical scale

Among the 13 CsCOL genes, 10 were found to be highly expressed in the leaf tissue, $\mathrm{Cs} C O \mathrm{~L} 2$ and $\mathrm{Cs} C O \mathrm{~L} 3$ were highly expressed in the female flower, and different expression patterns were found for CsCOL13, with its highest expression level in the stem, and lower expression levels in other tissues (Fig. 6).

Previous studies found that the $C O L$ gene played an important role in the regulation of flowering time. To evaluate the possible functions of $\mathrm{Cs} C O L$ genes, qRTPCR was used to analyze the expression levels of $C s C O L$ genes under different photoperiod treatments at $4 \mathrm{~h}$ intervals (Fig. 7). Under SD conditions, the diurnal expression pattern of the $C s C O L$ genes varied. The expression patterns were roughly divided into three types (Fig. 7). The first type exhibited high levels of expression at the end of darkness, including CsCOL1-3, CsCOL5-7 and CsCOL10-12. The second type showed an increased expression at 04:00 in the night (darkness), including CsCOL4, CsCOL8 and CsCOL13. The remaining CsCOL genes displayed highest expression at the end of light (Fig. 7). Under LD conditions, although the transcript level of all $C O L$ genes was induced in light, two types of diurnal expression patterns were observed (Fig. 7). The first type included the transcript levels of most $\mathrm{CsCOL}$ genes, which peaked at 12:00 PM in the day (light) (CsCOL1-5, CsCOL7, CsCOL8, CsCOL10, CsCOL11, and CsCOL13), while the second category exhibited the highest expression levels at 16:00 in the day (light) (CsCOL6, CsCOL9, and CsCOL12). Collectively, these results suggest that the majority of the $C s C O L$ genes exhibited a diurnal oscillation expression pattern under the SD and LD conditions.

To further explore the function of $C O L$ genes in cannabis, 2 early-flowering varieties, "Qingma 1" ("Q1") and "H7", and 2 late-flowering varieties, "Yunma 7" ("Y7") and "BM", selected from 126 accessions growing under short days, were using for investigating the expression patterns of 13 CsCOL genes. The flowering time of "Q1" and "H7" is $29 \mathrm{~d}$ and $31 \mathrm{~d}$ after sowing, respectively, while those of "Y7" and "BM" is $117 \mathrm{~d}$ and $113 \mathrm{~d}$, under SD conditions in the field (Fig. S1B). Under SD conditions, among the $13 \mathrm{CsCOL}$ genes, 2 genes (CsCOL4, and $C s C O L 11$ ) showed higher expression levels in these 2 early-flowering varieties than in these 2 late-flowering varieties at the peak of transcription levels, while 4 genes (CsCOL6, CsCOL7, CsCOL9, and CsCOL12) showed an opposing pattern. The remaining $C O L$ genes exhibited similar expression levels between these 4 varieties (Fig. 8).

\section{Discussion}

The $C O L$ gene family plays a key role in the regulation of flowering time, and has been reported in many plant species, including Arabidopsis, rice, maize, soybean (Glycine max), tomato (Solanum lycopersicum), and Populus, Lilium $\times$ formolongi $[5,10,12-14,27,28]$. However, a genome-wide investigation of the $C O L$ family gene in cannabis has yet to be conducted. Due to the 


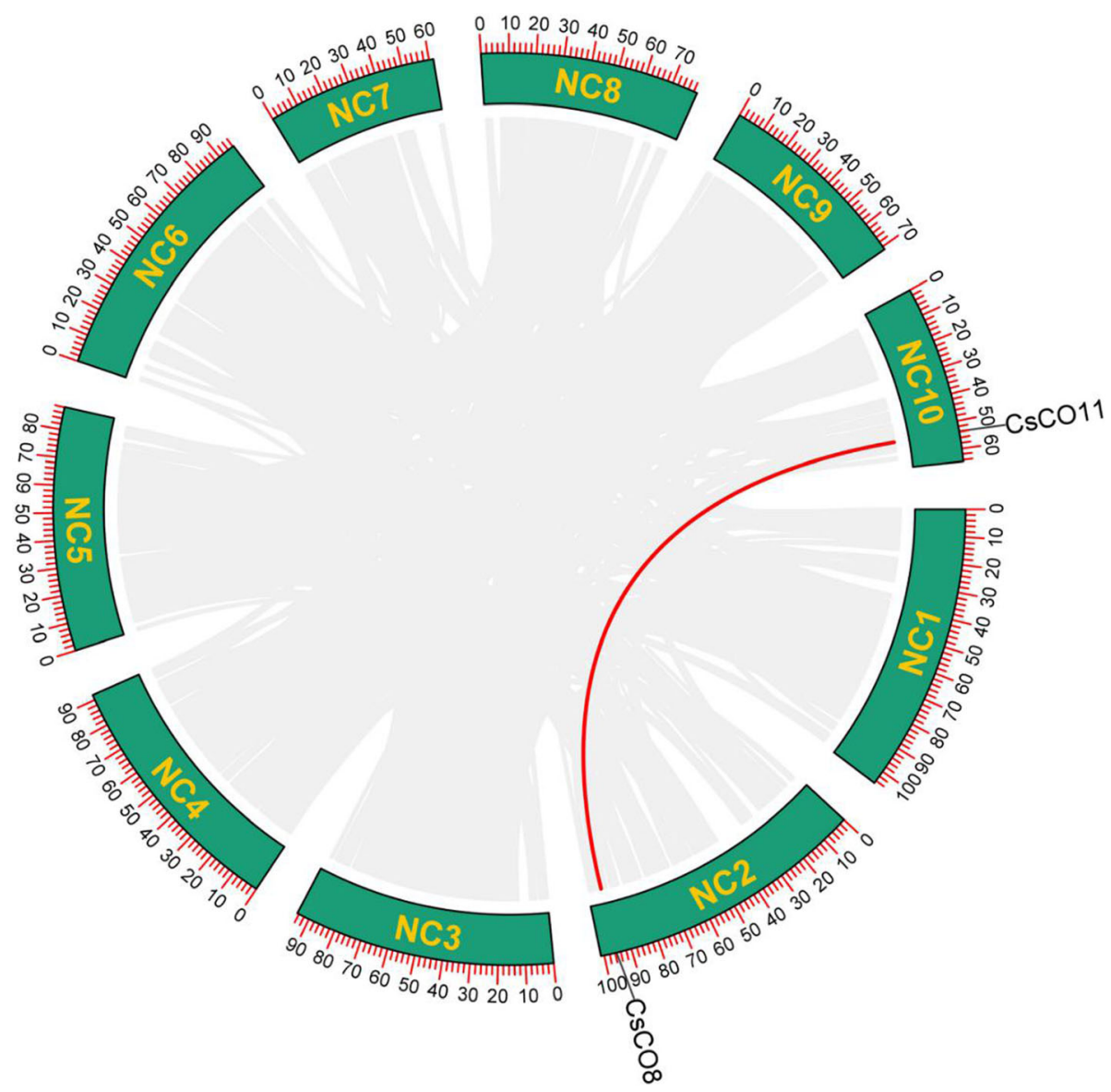

Fig. 4 Schematic representation of the interchromosomal relationships between the CSCOL genes in the cannabis genome. Coloured lines indicates the colinear gene pair, grey lines indicate the syntenic blocks in the cannabis genome

unavailability of a high-quality cannabis genome sequence, work on the genome-wide identification of $\mathrm{COL}$ genes in the cannabis genome has been lacking. The most recently assembled cannabis genome contained gene location information at the chromosome level [29], which allowed for a comprehensive analysis of the $C O L$ gene family in cannabis. In the present study, $13 \mathrm{COL}$ members were identified in the cannabis genome. These were divided into 3 subgroups (Figs. 1 and 2), which is similar to the grouping in rice and Arabidopsis [16]. Similar numbers of $C O L$ genes were found in other plants, including Populus (14 COL genes), sorghum (Sorghum bicolor L.) (15), and rice (16) [13, 16, 30]. The fact that the size of the genome of the four plants differed suggests that the number of $C O L$ genes in the $C O L$ superfamily was stable and did not vary with genome size.

Tandem replication events are associated with the occurrence of novel functions and gene expansion. In cannabis, the replication events have been found to occur in the CBCAS, THCAS, and CBDAS genes [29, 31]. On the other hand, no tandem duplication events have been observed in the COL genes of cotton [30]. However, in this study, a tandem gene pair (CsCOL6 and CsCOL12) was found on chromosome 3 in cannabis, indicating that gene replications may be an important driving force of cannabis gene evolution. In addition to tandem replication events, segmental duplication has been reported as the main driving force of gene expansion in the COL-like gene family in Gossypium and maize [12, 30]. Consistent with these findings, in this study, a segmental duplication gene pair (CsCOL8/CsCOL11) was found in the cannabis genome (Fig. 4). However, among the genes involved in duplication, this pair of duplication genes displayed different expression patterns under SD and LD conditions (Figs. 1, 7, and 8), which indicated that these genes experienced functional divergence during gene duplication. In addition, 15 pairs of orthologous genes between cannabis and cotton Raymond were identified, while only 2 pairs were found between cannabis and rice (Fig. 5). This observation implies that cannabis $C O L$ genes have a closer relationship with cotton Raymond than with rice, which may be consistent with the evolutionary relationship between monocotyledons and 


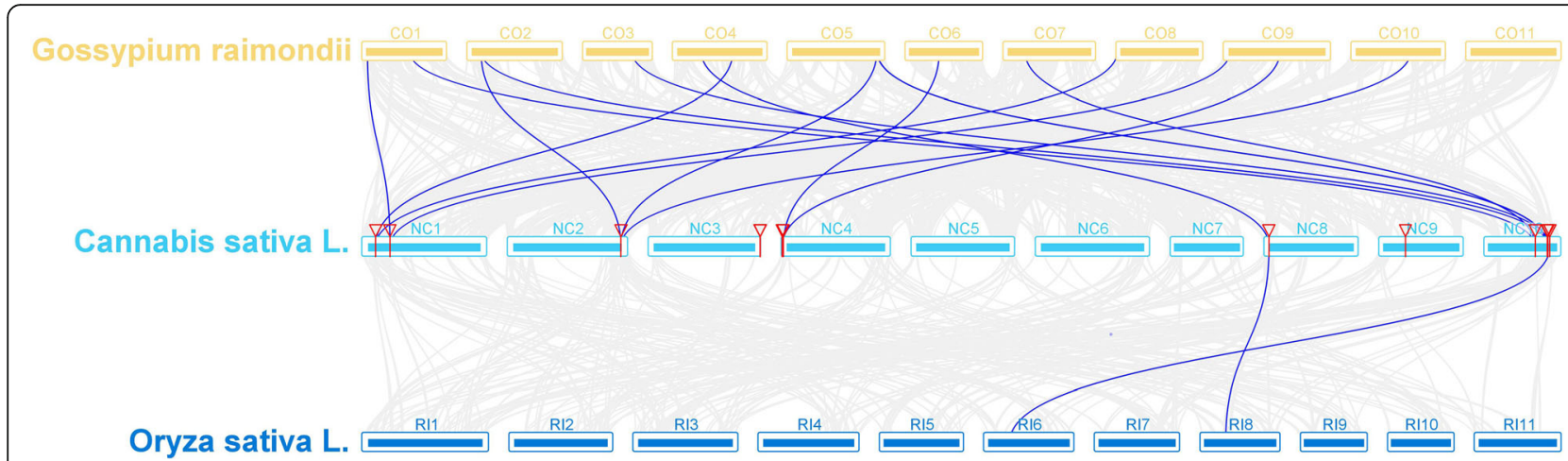

Fig. 5 Synteny analysis of the COL genes between cannabis and 2 representative species. Coloured lines highlight the colinear gene pair, while grey lines indicate the syntenic blocks within cannabis and other plant genomes

dicotyledons. Interestingly, CsCOL4 and CsCOL11 were found in both rice and cotton Raymond, indicating that these $C O L$ genes expanded in a species-specific manner from common ancestral genes before the dicot-monocot divergence.

Although previous studies have shown that $C O L$ genes are widely expressed in different plant tissues, they have been found to be preferentially expressed in the leaves $[5,14,17]$. Leaves sense photoperiod signals and express $C O L$ to activate FT and promote flowering [9]. In this study, we investigated the transcript levels of $13 \mathrm{CsCOL}$

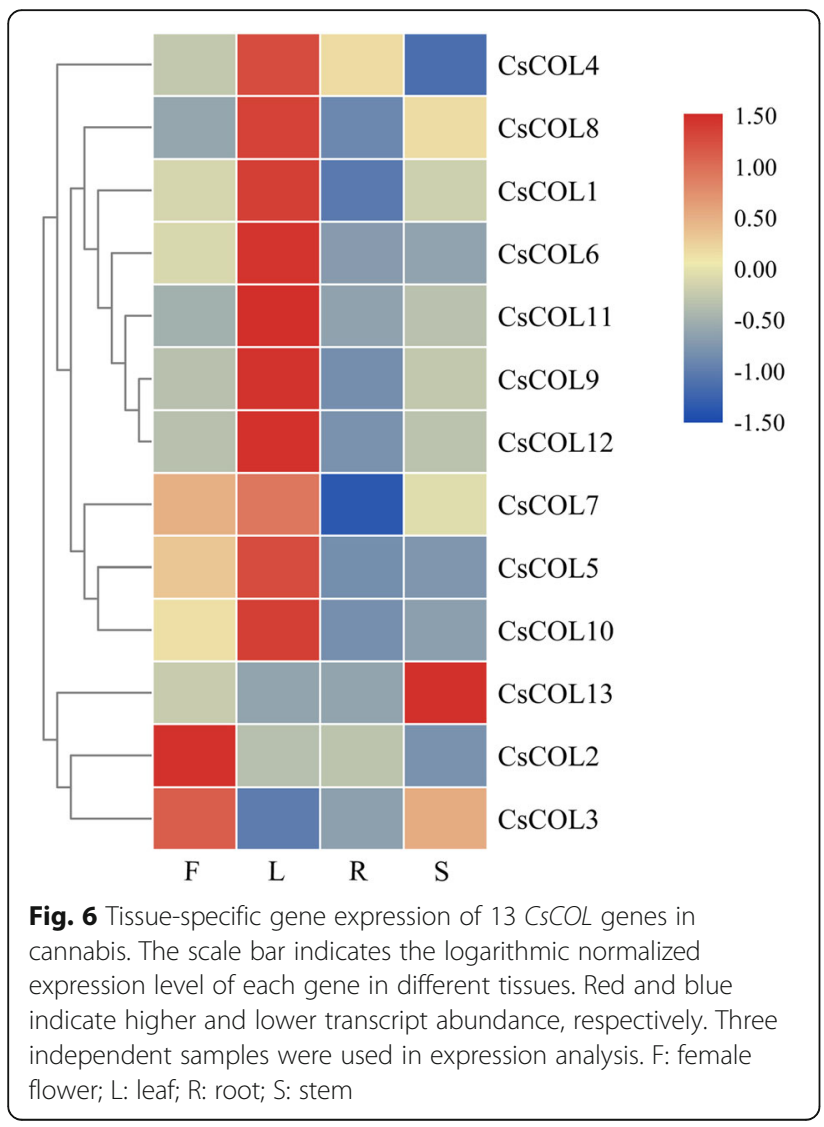

genes in various plant organs, including the female flower, leaves, roots, and stems. As a result, $10 \mathrm{COL}$ genes in cannabis were found to be preferentially expressed in the leaves, with an expression pattern similar to that observed in other plants, indicating their potential functions (Fig. 7).

The photoperiod is considered to be a key determining factor of flowering timing in plants, and $C O L$ genes have been demonstrated to be involved in the regulation of photoperiod-mediated flowering $[5,9,17]$. Therefore, we investigated the diurnal variations in the transcript levels of the CsCOL genes under LD and SD conditions. Under $\mathrm{SD}$ conditions, all the $C O L$ genes exhibited a diurnal oscillation expression pattern, with few differences between them. The transcript levels of $9 \mathrm{COL}$ gene members were found to peak at dawn (Fig. 7), similar to OsCOL16, PtCOL1/2, AtCOL1, AtCOL2, and AtCO in other plants [5, 13]. Under LD conditions, the expression patterns of all CsCOL genes were roughly divided into 2 types. The first type included $10 \mathrm{CsCOL}$ genes that were expressed more highly after light treatment, peaking at $12 \mathrm{~h}$, consistent with $\mathrm{COL}$ genes including PtCOL14 in Populus [13]. Similar to LfCOL13-16, OsCOL10, and OsCOL16, the remaining $3 \mathrm{CsCOL}$ genes showed higher expression levels in light than in darkness, and peaked at $16 \mathrm{~h}[5,14,17]$.

Different expression levels of $C O L$ genes may be associated with the flowering time in different varieties. In this study, the "Q1" and "H7" variety exhibited an earlier flowering time than "Y7" and "BM" under SD conditions in the field (Fig. S1B). To further determine the potential functions of $C S C O L$ genes in the regulation of flowering time, we evaluated the transcript levels of all CsCOL genes in the "Q1"、"H7"、 "BM" and "Y7" varieties under SD conditions. As shown in Fig. 8, the expression levels of CsCOL4 and $\mathrm{CsCOL8}$ were higher in the two early-flowering varieties ("Q1" and "H7") than the two late-flowering ("Y7" and "BM") at the peak transcript level, while six genes (CsCOL6, CsCOL7, CsCOL9, and 

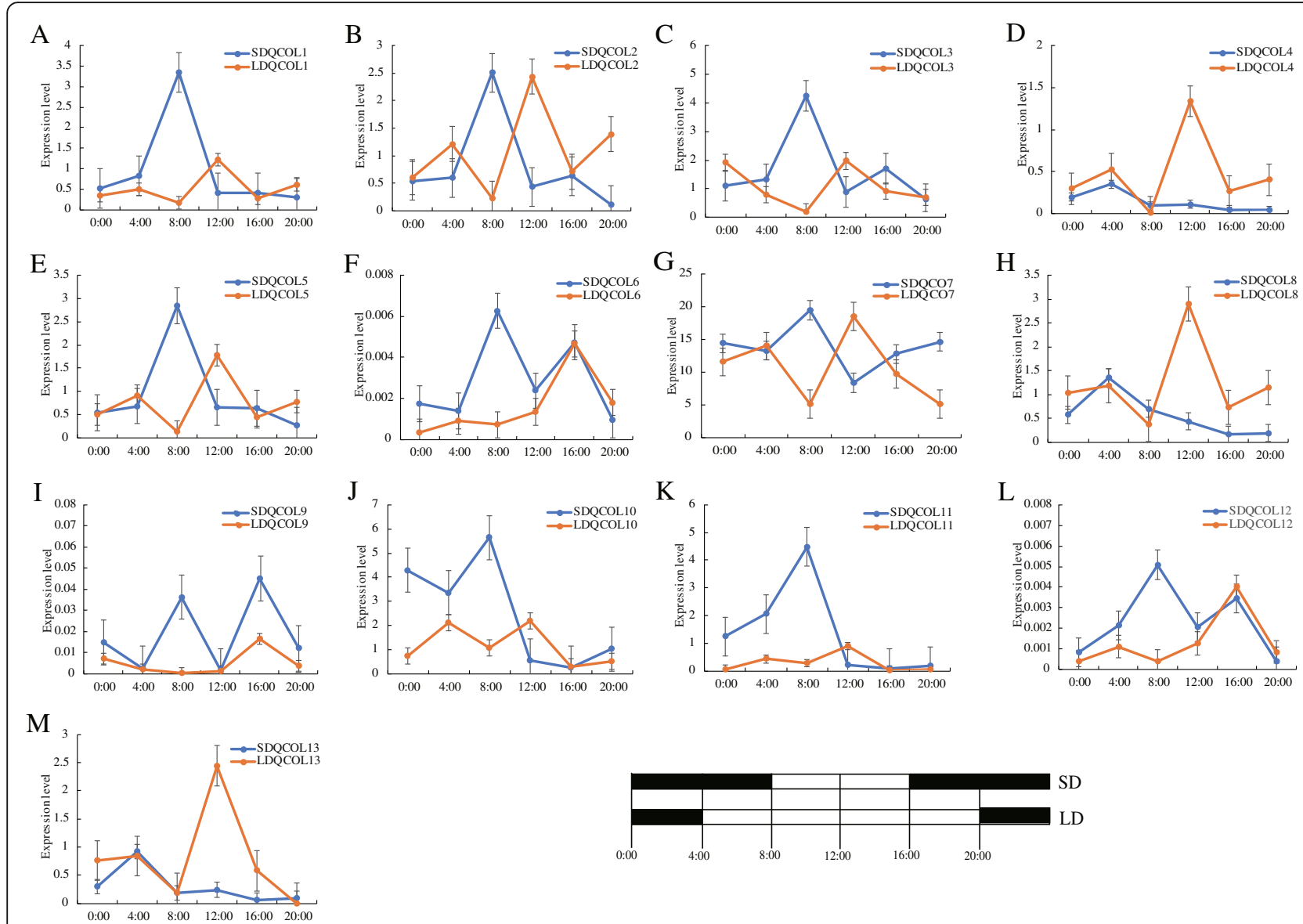

Fig. 7 Expression patterns of the CSCOL genes under LD ( $8 \mathrm{~h}$ dark/16 h light) and SD (16 h dark/8 $\mathrm{h}$ light) conditions. SDQCOL $1-13$ represents the expression levels of CSCOLs of "Qingma 1" under SD conditions; LDQCOL1-13 represents the expression levels of CsCOLs of "Qingma 1" under LD conditions. Black rectangle represented the darkness, while white rectangle represented the light. Data shown as mean $( \pm$ SD), $n=3$

CsCOL12) showed a contrasting pattern (Fig. 8). Interestingly, except for the similar expression patterns of CsCOL6, CsCOL12, and OsCOL16 under SD and LD conditions (Fig. 7) [13], these 3 genes also belonged to the same subgroup based on their phylogenetic relationship analysis (Fig. 2). In a previous study, CsCOL6 was found to repress flowering in rice [5]. Thus, CsCOL6/ CsCOL12 may exert a similar function to OsCOL16 in cannabis. However, this requires further study.

Previous studies have suggested that differences in the amino acid sequences of $C O L$ genes could explain their varied functions in the photoperiod-mediated flowering pathway $[5,10,20]$. In the present study, differences in the amino acid sequences of $\mathrm{CsCOL3}$ and $\mathrm{CsCOL7}$ were observed between "Q1" and "Y7", an early- and a lateflowering variety, respectively (Fig. S1 and Fig. S2). Moreover, differences were observed in the amino acid sequence of $\mathrm{CsCOL7}$ within the B-box, a conserved domain known for its functions in protein-protein interactions. However, whether these changes affect this type of function will need to be studied further.

\section{Conclusions}

To summarize, this study is the first to provide a comprehensive analysis of the $C O L$ gene family in cannabis. Our aim was to elucidate the evolution, expression profiles, and potential functions of these genes in the regulation of flowering in cannabis. Although the possible functions of the $C s C O L$ gene family require further study for validation, the systemic analysis conducted in this study provides a foundation for future studies on the biological and molecular functions of $C O L$ genes in cannabis.

\section{Methods}

Identification and analysis of physical and chemical properties of $\mathrm{CsCOL}$ gene family members in cannabis

The sequences of 17 Arabidopsis CONSTANS-like proteins were downloaded from the Arabidopsis Information Resource (TAIR) (http://www.aabidopsis.org/). The cannabis genome file and genome annotation file (assembly number: GCA_900626175.2) were obtained from the NCBI database (https://www.ncbi.nlm.nih.gov/) [29]. 


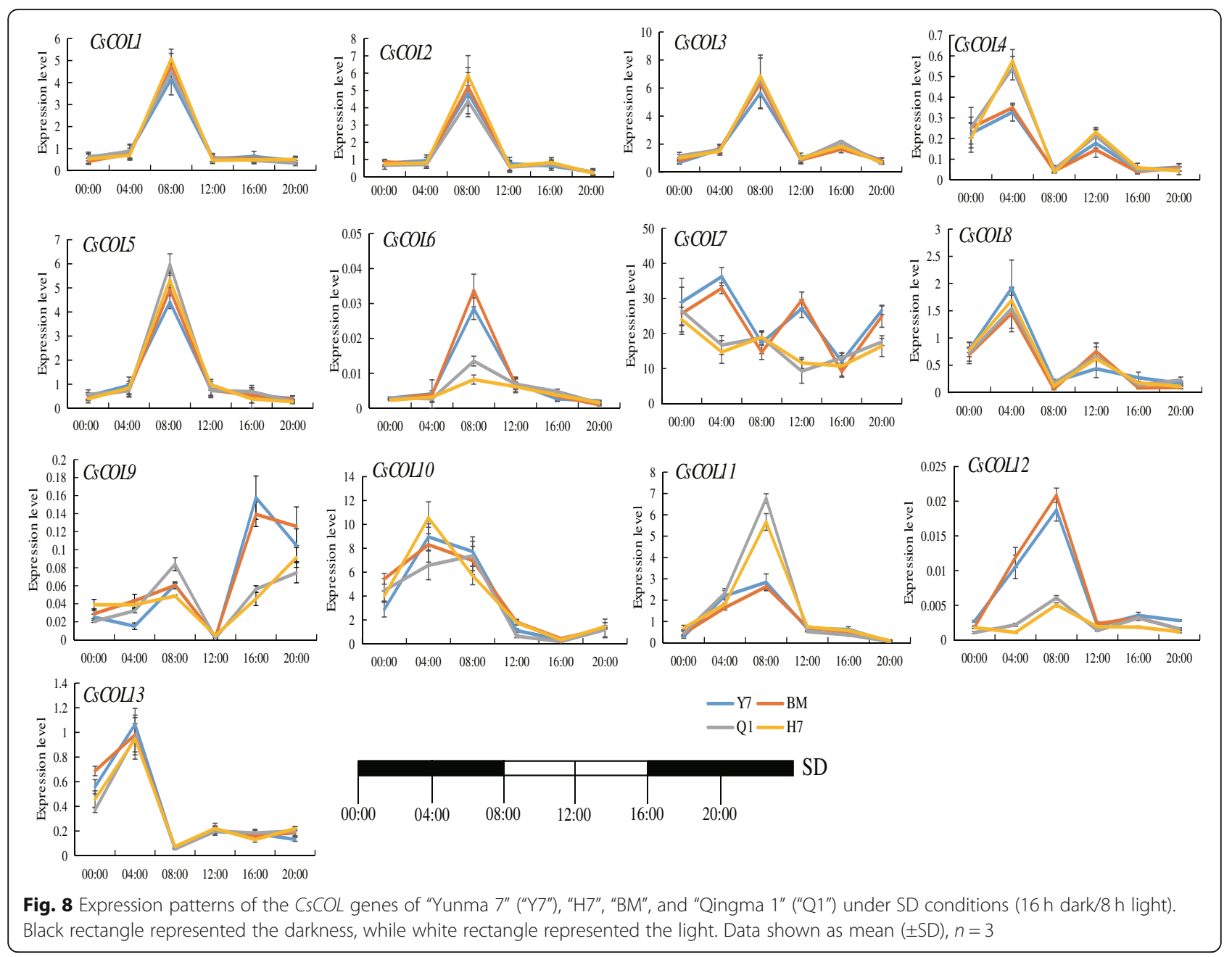

The software TBtools was used to compare the Arabidopsis $C O L$ gene with the cannabis genome by blast sequence alignment $\left(\mathrm{E}\right.$-value $\left.<1 \mathrm{E}^{-5}\right)$ and to screen the Cs $C O L$ family candidate genes in the cannabis genome. Next, the candidate genes were submitted to the Uniprot database (https://www.uniprot.org/) for batch comparison to verify whether they contained both CCT and Bbox conserved domains. ProtParam (http://web.expasy. org/protparam/) was used to analyse various physicochemical parameters of the $\mathrm{Cs} C O L$ genes.

\section{Gene cloning}

The primers pairs of the $C s C O L$ genes were designed according to the CDS sequences (Supplementary Table S1). cDNA of "Y7" and "Q1" was used as a template for each gene. PCR was performed as follows: an initial step at $94{ }^{\circ} \mathrm{C}$ for $5 \mathrm{~min}$, followed by 30 cycles of $30 \mathrm{~s}$ at $98^{\circ} \mathrm{C}$, $30 \mathrm{~s}$ at $55^{\circ} \mathrm{C}, 2 \mathrm{~min}$ at $68^{\circ} \mathrm{C}$, and a final extension of 10 min at $68^{\circ} \mathrm{C}$. After the PCR procedure was finished, the PCR product was purified, ligated to pGEM-T Easy vector, and transformed into E. coli DH5a. Positive clones were selected for sequencing. A list of the primers used for gene cloning is provided in Supplementary Table S1.

Multisequence alignment, phylogenetic analysis of $\mathrm{CsCOL}$ proteins, and gene structure analysis of $\mathrm{CsCOL}$

Multisequence alignment analysis of Arabidopsis, rice, and cannabis COL proteins was performed using Clustal X2.1 with the default parameters [32]. A phylogenetic tree was constructed using MEGA7.0 using the neighbour-joining (NJ) method. Bootstrap values $(>50 \%)$ were estimated using 1000 replicates. FigTree software was use to edit the phylogenetic tree. The protein structure of CsCOL was predicted using NCBI-CDD software online (https://www.ncbi.nlm.nih.gov/cdd/) with the default parameters (E-value $<0.01$ ). The conserved motif (Motif) of the CsCOL genes was analysed using MEME software online (http://meme-suite.org), and the predicted number was set to 10 . The coding sequence (CDS) and untranslated region (UTR) of $C s C O L$ were extracted from the cannabis genome annotation file using TBtools, which was also used to combine 
evolutionary tree, gene conservative motif, CDS, and UTR to construct a diagram to compare the evolutionary relationships and structures of $C s C O L$.

\section{Chromosome distribution and synteny analysis of CsCOL} Information on the chromosome location of the CsCOL genes was extracted from the cannabis genome file and gene annotation file using TBtools. Next, the physical location of CsCOL genes on chromosomes was constructed using TBtools. TBtools, MCscanX, and Circos were used to calculate and draw the tandem repeats of $C O L$ on the chromosome, the collinear genes among the cannabis genome, and among different species.

Evaluation of flowering time and photoperiod treatment "Yunma 7" ("Y7"), "BM", "H7", and“Qingma 1" (“Q1") were collected from the Institute of Bast Fibre Crops, China Academy of Agriculture Science, Changsha, China. The 2 varieties were randomly planted under natural short-day conditions in Changsha (southern China, $112^{\circ} 58^{\prime} \mathrm{E} / 28^{\circ} 11^{\prime} \mathrm{N}$, day length $<12 \mathrm{~h}$ during vegetative period). Once over $50 \%$ of the plants of each cultivar had bloomed, the flowering time was scored. For different photoperiod treatments under LD ( $16 \mathrm{~h}$ light $/ 8 \mathrm{~h}$ dark) and SD ( $8 \mathrm{~h}$ light $/ 16 \mathrm{~h}$ dark) conditions, the leaves of these seedlings were collected at 0:00, 04:00, 08:00, 12:00, 16:00, and 20:00 after photoperiod treatment. The resulting materials were promptly transferred into liquid nitrogen for RNA extraction, repeated independently in triplicate.

\section{RNA extraction and qRT-PCR analysis}

Total RNA was extracted from various tissues and leaves under different photoperiods using an RNAprep Pure Plant Kit (Tiangen, Beijing). The cDNA was synthesizing using a PrimeScript 1st Strand cDNA Synthesis Kit (TaKaRa, Japan). According to the manufacturer's instructions, quantitative RT-PCR (qRT-PCR) was conducted using a SYBR Premix Ex TaqTM kit (TaKaRa) on a 7500 Sequence Detection System (Applied Biosystems, USA). The DHS2 gene was amplified as the internal control. The primers used for qRT-PCR analysis are listed in Supplementary Table S2. The experiment was performed in triplicate.

\section{Abbreviations}

COL: CONSTANT-like; CBD: Cannabinoid; LD: Long day; SD: Short day; FT: FLOWERING LOCUS T; WGD: Whole genome duplication; CBCAS: CBCA synthases; THCAS: THCA synthases; CBDAS: CBDA synthases; qRTPCR: quantitative real-time PCR

\section{Supplementary Information}

The online version contains supplementary material available at https://doi. org/10.1186/s12870-021-02913-x.
Additional file 1: Table S1. The primers used for gene cloning in this study

Additional file 2: Table S2. The primers used for qRT-PCR in this study Additional file 3: Fig. S1. Comparison of flowering time between "Qingma 1", "Yunma 7", "H7", and "BM"

Additional file 4: Fig. S2. Coning of $\mathrm{CSCOL3}_{3}$ and $\mathrm{CSCOL7}$ from "Yunma 7"("Y7") and"Qingma 1"("Q1")

Additional file 5: Fig. S3. Comparison of the amino acid sequences of CsCOL3 (A) and CsCOL7 (B) between "Yunma 7 (Y7)" and "Qingma 1 $(\mathrm{Q} 1)^{\prime \prime}$

\section{Acknowledgements}

We thank all the staff in our lab for providing useful suggestions and technical assistance. We are very grateful to the editor and reviewers for providing constructive comments for the improvement of our manuscript.

Authors' contributions

G. P., and Z. L performed the experiments; G. P wrote the paper; M.Y., and J.T., participated in the sample collection and RNA extraction; A.-G.C., J.-J.L., H.-J.T., L. C and Y. D conducted bioinformatic analysis. S.-Q.H. revised the manuscript; L.-N.Z., and D.-F.L designed the experiment, and L.-N.Z also provided the materials. All authors read and approved the version to be published. All authors have read and agreed to the published version of the manuscript.

\section{Funding}

This research was supported by China Agriculture Technology Research System (CARS-16-E-02), Central Public-interest Scientific Institution Basal Research Fund (1610242020003, 1610242019001). Funds were used for performing the experiment, and analysis data and in writing the manuscript.

\section{Availability of data and materials}

The datasets for supporting the conclusions of this article are listed in the article and its additional files.

All coding sequences of CSCOL3 and CsCOL7 in "Qingma1" and "Yunma7" has been uploaded to the NCBI SRA database. SRA accession: PRJNA704531. The data will be accessible with the following link: "https://www.ncbi.nlm.nih. gov/sra/PRJNA704531".

\section{Declarations}

Ethics approval and consent to participate

Not applicable.

\section{Consent for publication}

Not applicable.

\section{Competing interests}

The authors declare that they have no competing interests.

\section{Author details}

${ }^{1}$ Institute of Bast Fiber Crops, Chinese Academy of Agricultural Sciences, Changsha 410205, China. ${ }^{2}$ Key Laboratory of the Biology and Process of Bast Fiber Crops, Ministry of Agriculture, Changsha, China.

Received: 5 November 2020 Accepted: 3 March 2021

Published online: 17 March 2021

References

1. Andre CM, Hausman JF, Guerriero G. Cannabis sativa: the Plant of the Thousand and one Molecules. Front Plant Sci. 2016;7:19.

2. Corroon J, Phillips JA. (2018). A cross-sectional study of cannabidiol users. Cannabis Cannabinoid Res. 2018;3(1):152-61.

3. Lisson SN, Mendham NJ, Carberry PS. Development of a hemp (Cannabis sativa L.) simulation model 2. The flowering response of two hemp cultivars to photoperiod. Aust J Exp Agric. 2000;40(3):413-7.

4. Song $\mathrm{YH}$, Ito $\mathrm{S}$, Imaizumi T. Flowering time regulation: photoperiod- and temperature-sensing in leaves [J]. Trends Plant Sci. 2013;18(10):575-83. 
5. Wu W, Zheng XM, Chen D, Zhang Y, Ma W, Zhang H, et al. OsCOL16, encoding a CONSTANS-like protein, represses flowering by up-regulating Ghd7 expression in rice. Plant Sci. 2017;260:60-9.

6. Liu J, Cheng Z, Li X, Xie L, Bai Y, Peng L, Li J, Gao J. Expression analysis and regulation network identification of the CONSTANS-like gene family in Moso bamboo (Phyllostachys edulis) under photoperiod treatments. DNA Cell Biol. 2019;38(7):607-26.

7. Imaizumi T, Schultz T, Harmon F, et al. FKF1F-BOX protein mediates cyclic degradation of a repressor of CONSTANS in Arabidopsis. Science. 2005; 309(5732):293-7.

8. Kobayashi D. Weigel, move on up, it's time for change-mobile signals controlling photoperiod-dependent flowering. Genes Dev. 2007;21:2371-84.

9. Turck F, Fornara F, Coupland G. Regulation and identity of florigen: FLOWERING LOCUS T moves center stage. Annu Rev Plant Biol. 2008;59: 573-94.

10. Robson F, Costa MM, Hepworth SR, et al. Functional importance of conserved domains in the flowering-time gene CONSTANS demonstrated by analysis of mutant alleles and transgenic plants. Plant J. 2001;28(6):619-31.

11. Khanna R, Kronmiller B, Maszle DR, Coupland G, Holm M, Mizuno T, et al. The Arabidopsis Bbox zinc finger family. Plant Cell. 2009;21:3416-20.

12. Song N, Xu Z, Wang J, Qin Q, Jiang H, Si W, Li X. Genome-wide analysis of maize CONSTANS-LIKE gene family and expression profiling under light/dark and abscisic acid treatment. GENE. 2018;673:1-11.

13. Li J, Gao K, Yang X, Khan WU, Guo B, Guo T, An X. Identification and characterization of the CONSTANS-like gene family and its expression profiling under light treatment in Populus. Int J Biol Macromol. 2020;161: 999-1010.

14. Li Y, Zhao Y, Zhang M, Jia G, Zaccai M. Functional and evolutionary characterization of the CONSTANS-like family in Liliumï̈1/2formolongi. Plant Cell Physiol. 2018;59(9):1874-88

15. Hu T, Wei Q, Wang W, Hu H, Mao W, Zhu Q, Bao C. Genome-wide identification and characterization of CONSTANS-like gene family in radish (Raphanus sativus). PLoS One. 2018;13(9):e204137.

16. Griffiths S, Dunford RP, Coupland G, Laurie DA. The evolution of CONSTANSlike gene families in barley, rice, and Arabidopsis. Plant Physiol. 2003;131: 1855-67.

17. Tan J, Jin M, Wang J, Wu F, Sheng P, Cheng Z, Wang J, Zheng X, Chen L, Wang M, Zhu S, Guo X, Zhang X, Liu X, Wang C, Wang H, Wu C, Wan J. OsCOL10, a CONSTANS-Like gene, functions as a flowering time repressor downstream of Ghd7 in Rice. Plant Cell Physiol. 2016;57(4):798-812.

18. Takase T, Kakikubo Y, Nakasone A, Nishiyama Y, Yasuhara M, Tokioka-Ono Y, Kiyosue T. Characterization and transgenic study of CONSTANS-LIKE8 (COL8) gene in Arabidopsis thaliana: expression of 35S: COL8 delays flowering under long-day conditions. Plant Biotechnol. 2011;28:439-66.

19. Wang H, Zhang Z, Li H, Zhao X, Liu X, Ortiz M, Lin C, Liu B. CONSTANS-LIKE 7 regulates branching and shade avoidance response in Arabidopsis. J Exp Bot. 2013;64(4):1017-24.

20. Yano M, Katayose Y, Ashikari M, Yamanouchi U, Monna L, Fuse T, Baba T, Yamamoto K, Umehara Y, Nagamura Y, et al. Hd1, a major photoperiod sensitivity quantitative trait locus in rice, is closely related to the Arabidopsis flowering time gene CONSTANS. Plant Cell. 2000;12(12):2473-84.

21. Sheng P, Wu F, Tan J, Zhang H, Ma W, Chen L, Wang J, Wang J, Zhu S, Guo $X$, et al. A CONSTANS-like transcriptional activator, OsCOL13, functions as a negative regulator of flowering downstream of OsphyB and upstream of Ehd1 in rice. Plant Mol Biol. 2016:92(1-2):209-22.

22. Datta S, Hettiarachchi GH, Deng XW, Holm M. Arabidopsis CONSTANS-LIKE3 is a positive regulator of red light signaling and root growth. Plant Cell. 2006;18(1):70-84.

23. Hassidim M, Harir Y, Yakir E, et al. Over-expression of CONSTANS-LIKE 5 can induce flowering in short-day grown Arabidopsis. Planta. 2009;230(3):481-91.

24. Chaurasia AK, Patil HB, Azeez A, Subramaniam VR, Krishna B, Sane AP, et al. Molecular characterization of CONSTANS-Like (COL) genes in banana (Musa acuminata L. AAA Group, cv. Grand Nain). Physiol Mol Biol Plants. 2016:22:1-15.

25. Holefors A, Opseth L, Ree RA, Ripel L, Snipen L, Fossdal CG, Olsen JE. Identification of PaCOL1 and PaCOL2, two CONSTANS-like genes showing decreased transcript levels preceding short day induced growth cessation in Norway spruce. Plant Physiol Biochem. 2009;47(2):105-15.

26. Ding J, Böhlenius $H$, Rühl MG, Chen P, Sane S, Zambrano JA, et al. GIGA NTEA-like genes control seasonal growth cessation in Populus. New Phytol. 2018;218:1491-503.
27. Yang $T$, He Y, Niu S, Yan S, Zhang Y. Identification and characterization of the CONSTANS (CO)/CONSTANS-like (COL) genes related to photoperiodic signaling and flowering in tomato. Plant Sci. 2020;301:110653.

28. Mengarelli DA, Zanor MI. Genome-wide characterization and analysis of the CCT motif family genes in soybean (Glycine max). PLANTA. 2021;253(1):15.

29. Laverty KU, Stout JM, Sullivan MJ, Shah H, Gill N, Holbrook L, Deikus G, Sebra R, Hughes TR, Page JE, et al. A physical and genetic map of Cannabis sativa identifies extensive rearrangements at the THC/CBD acid synthase loci. Genome Res. 2019;29(1):146-56.

30. Qin W, Yu Y, Jin Y, Wang X, Liu J, Xi J, Li Z, Li H, Zhao G, Hu W, et al. Genome-wide analysis elucidates the role of CONSTANS-like genes in stress responses of cotton. Int J Mol Sci. 2018;19(9):2658.

31. Weiblen GD, Wenger JP, Craft KJ, ElSohly MA, Mehmedic Z, Treiber EL, Marks MD. Gene duplication and divergence affecting drug content in Cannabis sativa. New Phytol. 2015;208(4):1241-50.

32. Larkin M, Blackshields G, Brown N, Chenna R, McGettigan P, Larkin MA, Blackshields G, Brown NP, Chenna R, McGettigan PA, McWilliam H, Valentin F, Wallace IM, Wilm A, Lopez R, et al. Clustal W and Clustal X version 2.0. Bioinformatics. 2007;23(21):2947-8.

\section{Publisher's Note}

Springer Nature remains neutral with regard to jurisdictional claims in published maps and institutional affiliations.

Ready to submit your research? Choose BMC and benefit from

- fast, convenient online submission

- thorough peer review by experienced researchers in your field

- rapid publication on acceptance

- support for research data, including large and complex data types

- gold Open Access which fosters wider collaboration and increased citations

- maximum visibility for your research: over $100 \mathrm{M}$ website views per year

At BMC, research is always in progress.

Learn more biomedcentral.com/submissions 